\title{
One year into COVID-19 pandemic, what do we have to look forward to?
}

\author{
Ivan FN Hung *, MD, FRCP (Lond, Edin) \\ Department of Medicine, Queen Mary Hospital, Hong Kong \\ *Corresponding author: ivanhung@hku.hk
}

Hong Kong Med J 2021;27:92-4

https://doi.org/10.12809/hkmj215113

At the time of writing, Hong Kong has just experienced the end of a fourth wave of the coronavirus disease 2019 (COVID-19) pandemic after imposing stringent infection control measures. ${ }^{1}$ With the recent launch of the COVID-19 vaccination programme, we are finally seeing the light at the end of the tunnel. Nevertheless, it will take major efforts from the Hong Kong SAR Government and various parties to reach the herd immunity level in order to lift all infection control measures.

In 2003, there was a severe acute respiratory syndrome (SARS) outbreak in Hong Kong and mainland China. Between March and June 2003, 1750 patients were diagnosed to have SARS in Hong Kong, with 286 deaths. $^{2}$ Despite identification of the SARS coronavirus as the cause of SARS ${ }^{3}$ and description of the disease pathogenesis model by a team from The University of Hong Kong, ${ }^{4}$ it was a painful experience with hefty loss of life. The major outbreak in the Amoy Garden estate due to contamination of the sewage in the U-traps which subsequently led to the airborne transmission of the SARS infection among the estate residents highlighted the overcrowded living conditions in Hong Kong. ${ }^{5}$ By June 2003, 386 healthcare workers were diagnosed to have SARS and eight of them - four doctors, one nurse, and three healthcare assistantshad succumbed. ${ }^{3}$ The initial shortage of masks and protective clothing for healthcare personnel and the lack of negative pressure isolation facilities resulted in significant nosocomial transmission of the virus. The majority of the diagnoses were made clinically and radiologically, and patients were cohort in large general medical ward. Treatment options were limited to steroids and ventilator support for those who developed respiratory failure. Many patients who were fortunate to recover from the infection suffered from lung fibrosis and the crippling longterm adverse effects of high-dose steroids. ${ }^{6}$ The SARS outbreak in 2003 highlighted the lack of communication among the health authorities in Hong Kong, mainland China, and the rest of the world. The establishment of the Centre for Health Protection in Hong Kong has helped overcome this shortcoming. The introduction of negative pressure isolation facilities in all major public hospitals hugely reduces the risk of nosocomial transmission and allows infected patients to safely cohort with minimal environmental contamination. ${ }^{7}$

Benefitting from experiences with SARS in 2003 and the swine flu pandemic in 2009, the Department of Health in Hong Kong implemented intense surveillance measures including tight border restrictions, social distancing, and mask wearing in the community. ${ }^{8}$ Vigorous contact tracing by the Centre of Health Protection enabled early quarantine and isolation. Deep throat saliva sampling for diagnostic screening reduced the human resources needed while maintaining high sensitivity. ${ }^{9}$ Such measures resulted in a relatively low number of confirmed infections despite the Hong Kong's status as an international transport hub. The negative pressure isolation facilities in Hospital Authority hospitals allowed prompt isolation and treatment of patients with COVID-19 with moderate to severe disease and identified risk factors. The establishment of a community treatment facility at AsiaWorldExpo allowed the isolation of large number of confirmed patients with mild disease, thus relieving the pressure on isolation bed facilities. The recent establishment of an infection control centre at North Lantau Hospital will further increase the number of negative pressure beds available.

Various measures have been shown to reduce the complication rate and shorten hospital stay for patients with COVID-19. Early treatment from symptom onset with the triple combination of interferon beta-1b, lopinavir-ritonavir, and ribavirin resulted in significantly quicker clinical improvement and shorter duration of viral shedding in patients hospitalised with COVID-19. ${ }^{10}$ The choice of antivirals was based on results from previous in vitro and in vivo animal studies on repurposing drugs for treatment of SARS 2003, Middle East respiratory syndrome, and SARS-CoV-2. ${ }^{11,12}$ Remdesivir, low-dose dexamethasone, convalescent plasma, and other immunomodulatory therapies have also been used to treat patients with hyperinflammatory response. ${ }^{13-17}$ Regular clinical assessment of patients with COVID-19, with regular reverse transcription polymerase chain reaction or biochemical testing, as well as radiological imaging, allows for close monitoring and better prediction of the patient's progress and for guiding changes to the 
patient's treatment. Adopting the immunoglobulin $G$ seroconversion has also facilitated patient's discharge when clinically deem fit.

Looking to the future, safe and effective COVID-19 vaccines are required. At the time of writing (24 March 2021), more than 80 different vaccines are undergoing human clinical trials, and 13 have gained full approval or have been authorised for emergency use. ${ }^{18}$ The Hong Kong SAR Government has implemented a territory-wide programme to offer COVID-19 vaccinations free of charge for all Hong Kong residents. To ensure the safety and efficacy of the vaccines, the Food and Health Bureau and the Department of Health have set up an Expert Advisory Panel to the Chief Executive. The joint Scientific Committees on Emerging and Zoonotic Diseases and Vaccine Preventable Diseases also regularly review the scientific evidence and relevant data on COVID-19 vaccines procured by the Government, and provide recommendations on the population groups to receive the COVID-19 vaccines. The Expert Committee on Clinical Events Assessment Following COVID-19 Immunisation has been established to provide independent assessment of potential causal links between adverse events following immunisation with any of the COVID-19 vaccines and to provide expert advice to the Government on safety-related matters. The Hong Kong SAR Government has currently procured three different platforms of COVID-19 vaccine from different vendors, including Comirnaty ${ }^{19}$ (mRNA vaccine), CoronaVac ${ }^{20}$ (inactivated whole cell vaccine), and Oxford/AstraZeneca ${ }^{21}$ (ChAdOx1 adenovirus vector vaccine), which will provide a wide choice of COVID-19 vaccines with good safety and efficacy profiles from which Hong Kong residents can choose. The Government is also planning procurement of a fourth COVID-19 vaccine. With a low COVID-19 seroprevalence among the population in Hong Kong, a high COVID-19 vaccination rate will be important to reach the satisfactory herd immunity level to allow relaxation of infection control measures. ${ }^{22}$ Important data including the long-term safety, clinical efficacy and effectiveness, and neutralising antibody protection against the new SARS-CoV-2 variants (B.1.1.7, B.1.351 and P.1) will be essential to plan for future vaccination programmes. The frequency of COVID-19 vaccination will also depend on the rate of emergence of these new variants.

The lessons learned from the SARS outbreak in 2003 have helped to successfully limit the spread of COVID-19 within Hong Kong. Nevertheless, the global effort against the COVID-19 pandemic will require cooperation among governments, international organisations, research institutes, scientists, clinicians, and most important of all, individual citizens.

\section{Author contributions}

The author contributed to the editorial, approved the final version for publication, and takes responsibility for its accuracy and integrity.

\section{Disclosures}

IFN Hung is a member of the Advisory Panel on COVID-19 Vaccines; and the co-convener of the Expert Committee on Clinical Events Assessment Following COVID-19 Immunisation for the Hong Kong SAR Government.

\section{References}

1. Coronavirus disease (COVID-19) in HK. Available from: https://chp-dashboard.geodata.gov.hk/covid-19/en.html. Accessed 24 Mar 2021.

2. Lee SH. The SARS epidemic in Hong Kong: what lessons have we learned? JR Soc Med 2003;96:374-8.

3. Peiris JS, Lai ST, Poon LL, et al. Coronavirus as a possible cause of severe acute respiratory syndrome. Lancet 2003;361:1319-25.

4. Peiris JS, Chu CM, Cheng VC, et al. Clinical progression and viral load in a community outbreak of coronavirusassociated SARS pneumonia: a prospective study. Lancet 2003;361:1767-72.

5. Chu CM, Cheng VC, Hung IF, et al. Viral load distribution in SARS outbreak. Emerg Infect Dis 2005;11:1882-6.

6. Chan KS, Zheng JP, Mok YW, et al. SARS: prognosis, outcome and sequelae. Respirology 2003;8:S36-40.

7. Cheng VC, Wong SC, Chuang V, et al. Absence of nosocomial transmission of coronavirus disease 2019 (COVID-19) due to SARS-CoV-2 in the prepandemic phase in Hong Kong. Am J Infect Control 2020;48:8906.

8. Cowling BJ, Ali ST, Ng TW, et al. Impact assessment of non-pharmaceutical interventions against coronavirus disease 2019 and influenza in Hong Kong: an observational study. Lancet Public Health 2020;5:e279-88.

9. To KK, Tsang OT, Leung WS, et al. Temporal profiles of viral load in posterior oropharyngeal saliva samples and serum antibody responses during infection by SARSCoV-2: an observational cohort study. Lancet Infect Dis 2020;20:565-74.

10. Hung IF, Lung KC, Tso EY, et al. Triple combination of interferon beta-1b, lopinavir-ritonavir, and ribavirin in the treatment of patients admitted to hospital with COVID-19: an open-label, randomised, phase 2 trial. Lancet 2020;395:1695-704.

11. Yuan S, Chan CC, Chik KK, et al. Broad-spectrum hostbased antivirals targeting the interferon and lipogenesis pathways as potential treatment options for the pandemic coronavirus disease 2019 (COVID-19). Viruses 2020;12:628.

12. Chu CM, Cheng VC, Hung IF, et al. Role of lopinavir/ ritonavir in the treatment of SARS: initial virological and clinical findings. Thorax 2004;59;252-6.

13. Beigel JH, Tomashek KM, Dodd LE, et al. Remdesivir for the treatment of COVID-19-final report. N Engl J Med 2020;383:1813-26.

14. RECOVERY Collaborative Group; Horby P, Lim WS, et al. Dexamethasone in hospitalized patients with Covid-19. N Engl J Med 2021;384:693-704.

15. Libster R, Pérez Marc G, Wappner D, et al. Early high-titer 
plasma therapy to prevent severe Covid-19 in older adults. N Engl J Med 2021;384:610-8.

16. Guaraldi G, Meschiari M, Cozzi-Lepri A, et al. Tocilizumab in patients with severe COVID-19: a retrospective cohort study. Lancet Rheumatol 2020;2:e474-84.

17. Kalil AC, Patterson TF, Mehta AK, et al. Baricitinib plus remdesivir for hospitalized adults with Covid-19. N Engl J Med 2021;384:795-807.

18. World Health Organization. The COVID-19 candidate vaccine landscape and tracker. https:/www.who.int/ publications/m/item/draft-landscape-of-covid-19candidate-vaccines. Accessed 24 Mar 2021.

19. Dagan N, Barda N, Kepten E, et al. BNT162b2 mRNA Covid-19 vaccine in a nationwide mass vaccination setting. N Engl J Med 2021 Feb 14. Epub ahead of print.
20. Centre for Health Protection, Department of Health, Hong Kong SAR Government. Consensus interim recommendations on the use of CoronaVac in Hong Kong. https://www.chp.gov.hk/files/pdf/consensus_interim recommendations_on_the_use_of_coronavac_in_hk_as_ of_19_feb_2021.pdf. Accessed 24 Mar 2021.

21. Ramasamy MN, Minassian AM, Ewer KJ, et al. Safety and immunogenicity of ChAdOx $1 \mathrm{nCoV}-19$ vaccine administered in a prime-boost regimen in young and old adults (COV002): a single-blind, randomised, controlled, phase 2/3 trial. Lancet 2021;396:1979-93.

22. To KK, Cheng VC, Cai JP, et al. Seroprevalence of SARS-CoV-2 in Hong Kong and in residents evacuated from Hubei province, China: a multicohort study. Lancet Microbe 2020;1:e111-8. 